\title{
A NOTE ON THE DAMPING IN ROLL OF A CRUCIFORM WINGED BODY*
}

By JOHN W. MILES** (Fulbright Lecturer, Auckland University College, Auckland, New Zealand)

1. Introduction. In the following we apply the slender body theory of Ward ${ }^{1}$ to the calculation of the damping in roll of a slender body of revolution of radius $a$, bearing cruciform wings of total span $2 b$.

It is required to find a solution, $\phi(s, x, y)$, to Laplace's equation satisfying the boundary conditions

$$
\begin{array}{ll}
\left.\phi_{y}(s, x, y)\right|_{y=0}=p x, & a<|x|<b, \\
\left.\phi_{x}(s, x, y)\right|_{x=0}=-p y, & a<|y|<b, \\
\left.\phi_{r}(s, r \cos \theta, r \sin \theta)\right|_{r=a}=0, &
\end{array}
$$

where $(s, x, y)$ are a set of right handed, Cartesian coordinates with $s$ measured positive downstream from the body nose, and $p$ is the angular velocity about the $s$ axis. The rolling moment is then given by

$$
N=-\rho U \oint \phi(l, x, y)(x d x+y d y),
$$

where the integral is taken around the cross section at the trailing edge $(s=l)$, the latter being assumed to be straight and transverse the free stream. Whereas in Eq. (1) $a$ and $b$ are functions of $s$, exhibiting a monotonic increase therewith, we hereafter refer only to their values at $s=l$.

2. Solution for potential. The conformal transformation

$$
\begin{gathered}
\left(z+a^{2} / z\right)^{2}-\left(b+a^{2} / b\right)^{2}=\left(\zeta-c^{2} / \zeta\right)^{2}, \\
c^{2}=\frac{1}{2}\left(b^{2}+a^{4} / b^{2}\right)
\end{gathered}
$$

maps the profile of the cruciform winged body in the $z(=x+i y)$ plane on the circle $|\zeta|=c$ in the $\zeta$ plane, the wings appearing as four, symmetrically disposed arcs of subtended angle $2 \varphi_{0}$, where

$$
\cos 2 \varphi_{0}=(a / c)^{2}=2(a / b)^{2}\left[1+(a / b)^{4}\right]^{-1} .
$$

Transforming the boundary conditions (1.1) and carrying out the solution to the then classical problem, we obtain the potential on $\zeta=c e^{i \varphi}$ in the form

$$
\begin{array}{r}
\phi=-\left(2 p c^{2} / \pi\right) \sin 4 \varphi \int_{0}^{\varphi_{0}}\left[\left(\cos 2 \psi-\cos 2 \varphi_{0}\right)+\left(\cos ^{2} 2 \psi-\cos ^{2} 2 \varphi_{0}\right)^{1 / 2}\right] \\
\cdot(\cos 4 \psi-\cos 4 \varphi)^{-1} d \psi .
\end{array}
$$

Carrying out the required integrations in Eq. (4), we have

${ }^{*}$ Received August 21, 1951.

${ }^{* *}$ On leave from University of California, Los Angeles; formerly Consultant, U. S. Naval Ordnance Test Station, Inyokern, California. 


$$
\begin{aligned}
\phi=-\left(p c^{2} / 2 \pi\right) & \left\{\cos 2 \varphi \ln \left|\frac{\tan \left(\varphi+\varphi_{0}\right)}{\tan \left(\varphi-\varphi_{0}\right)}\right|-\cos 2 \varphi_{0} \ln \left|\frac{\sin 2\left(\varphi+\varphi_{0}\right)}{\sin 2\left(\varphi-\varphi_{0}\right)}\right|\right. \\
& +\mathbf{K}\left(\sin 2 \varphi_{0}\right) \sin 4 \varphi+2\left[\mathbf{E}\left(\sin 2 \varphi_{0}\right) \mathbf{F}\left(\sin ^{-1}\left(\sin 2 \varphi / \sin 2 \varphi_{0}\right), \sin 2 \varphi_{0}\right)\right. \\
& \left.\left.-\mathbf{K}\left(\sin 2 \varphi_{0}\right) \mathbf{E}\left(\sin ^{-1}\left(\sin 2 \varphi / \sin 2 \varphi_{0}\right), \sin 2 \varphi_{0}\right)\right]\left(\sin ^{2} 2 \varphi_{0}-\sin ^{2} 2 \varphi\right)^{1 / 2}\right\},
\end{aligned}
$$

where $\mathbf{K}$ and $\mathbf{E}$, with single arguments, denote complete elliptic integrals of the first and second kinds; respectively, and $\mathbf{F}$ and $\mathbf{E}$, with two arguments, denote the corresponding incomplete integrals of modulus $\sin 2 \varphi_{0}$ and amplitude $\sin ^{-1}(\sin 2 \varphi / \sin 2 \varphi)$. For the special case of no body $\left(a=0, \varphi_{0}=\pi / 4\right)$ Eq. (5) reduces to

$$
\phi=-\left(p b^{2} / 2 \pi\right) \cos 2 \varphi \ln \left|\frac{1+\sin 2 \varphi}{1-\sin 2 \varphi}\right| \quad(a=0)
$$

3. Rolling moment. The integrals subsequent to the substitution of Eq. (2.5) in Eq. (1.2) appear to be intractable, and it is expedient to proceed by substituting instead Eq. (2.4), which leads to

$$
\begin{aligned}
N / N_{0} & =(2 / \pi)^{2}\left[1+(a / b)^{4}\right]^{2} \sum_{1}^{\infty}\left(I_{n}^{2} / n\right), \\
N_{0} & =-\pi \rho U p b^{4} / 4, \\
I_{n} & =\int_{0}^{\varphi_{0}} \sin (4 n \varphi) d\left[\cos 2 \varphi+\left(\cos ^{2} 2 \varphi-\cos ^{2} 2 \varphi_{0}\right)^{1 / 2}\right] .
\end{aligned}
$$

The reference moment, $N_{0}$, is twice that acting on a single wing of (small) span $2 b$, so that $N / N_{0}$ represents an overall interference factor. For values of $(a / b)$ near unity the convergence of Eq. (1) is poor, but the potential may be expanded in powers of $\varphi_{0}$ to obtain

$$
N / N_{0}=8[1-(a / b)]^{2}\{1-1.57[1-(a / b)]\}+O\left\{[1-(a / b)]^{4}\right\}
$$

For the special case $a=0$, the substitution of Eq. (2.6) in Eq. (1.2) yields an interference factor of $\left(8 / \pi^{2}\right)$, which may be checked by summing Eq. (1), viz.

$$
N / N_{0}=(2 / \pi)^{2} \sum_{1}^{\infty}\left[\left(\frac{4 n}{4 n^{2}-1}\right)^{2} / n\right]=8 / \pi^{2}
$$

in agreement with the result obtained by Adams. ${ }^{2}$ We remark that the first three terms in Eq. (5) yield the correct result to better than $2 \%$, implying a satisfactorily rapid convergence of Eq. (1) for small $(a / b)$.

More complete numerical results are to be given in a forthcoming NOTS report, ${ }^{3}$ representing work supported by the Office of Naval Research.

\section{REFERENCES}

(1) G. N. Ward, Supersonic flow past slender pointed bodies, Q. J. Mech. Appl. Math. 2, 75-97 (1949).

(2) G. J. Adams, Theoretical damping in roll and rolling effectiveness of slender cruciform wings, NACA TN 2270 (1951).

(3) J. W. Miles, On the damping in roll of a slender cruciform wing body, USNOTS Report, Inyokern, California, (1951). 